\title{
Characterization of magnetic symmetry and electric polarization of $\mathrm{YCr}_{0.5} \mathrm{Fe}_{0.5} \mathrm{O}_{3}$
}

\author{
J. Y. Yang $\odot,{ }^{1,2}$ X. D. Shen, ${ }^{3,4}$ V. Pomjakushin, ${ }^{1}$ L. Keller, ${ }^{1}$ E. Pomjakushina, ${ }^{2}$ Y. W. Long, ${ }^{3,5}$ and M. Kenzelmann ${ }^{1, *}$ \\ ${ }^{1}$ Laboratory for Neutron Scattering and Imaging, Paul Scherrer Institut, CH-5232 Villigen, Switzerland \\ ${ }^{2}$ Laboratory for Multiscale Materials Experiments, Paul Scherrer Institut, CH-5232 Villigen, Switzerland \\ ${ }^{3}$ Beijing National Laboratory for Condensed Matter Physics, Institute of Physics, Chinese Academy of Sciences, Beijing 100190, China \\ ${ }^{4}$ School of Physics, University of Chinese Academy of Sciences, Beijing 100049, China \\ ${ }^{5}$ Songshan Lake Materials Laboratory, Dongguan, Guangdong 523808, China
}

(Received 16 April 2019; revised manuscript received 11 October 2019; published 13 January 2020)

\begin{abstract}
We investigate the magnetic structure and the electric properties of $\mathrm{YCr}_{0.5} \mathrm{Fe}_{0.5} \mathrm{O}_{3}$, which was earlier reported to feature ferroelectricity from nonequivalent spins due to the cation disorder. We find a centrosymmetric magnetic order at all temperatures below $T_{N}$, which is incompatible with magnetically induced ferroelectricity. Our dielectric and pyroelectric studies also do not support intrinsic ferroelectricity in this system.
\end{abstract}

DOI: 10.1103/PhysRevB.101.014415

\section{INTRODUCTION}

Multiferroic materials in which magnetism and ferroelectric order coexist have been attracting great attention due to both fundamental interests and the potential for numerous applications [1-6]. Based on the different origins of ferroelectricity, single-phase multiferroic materials can be classified into two categories: type I and type II [7]. TypeII multiferroics refer to those magnetic ferroelectrics where ferroelectricity is induced by some specific magnetic orders, as evidenced in $\mathrm{TbMnO}_{3}$ [8]. All other multiferroics are called the type-I multiferroics, in which ferroelectricity does not have magnetic origin, with $\mathrm{BiFeO}_{3}$ as an example [9].

As is well known, symmetry determines the physical properties of materials and the possible coupling terms between order parameters. The two basic symmetries associated with magnetic order and ferroelectricity are the time-reversal symmetry and the space-inversion symmetry, respectively. A multiferroic that is both ferromagnetic and ferroelectric thus possesses neither time-reversal symmetry nor space-inversion symmetry. The total symmetry of multiferroics is the product of the crystal symmetry and magnetic symmetry, so it is possible to generate multiferroicity in a centrosymmetric crystal lattice if its magnetic structure breaks the space-inversion symmetry $[10,11]$. For type-II multiferroics, the presence of competing interactions and/or magnetic frustration induces complex magnetic order that breaks inversion symmetry, thus allowing ferroelectricity to emerge $[8,12]$.

In this work, we focus on the compound $\mathrm{YCr}_{0.5} \mathrm{Fe}_{0.5} \mathrm{O}_{3}$ which belongs to the larger $\mathrm{YCr}_{1-x} \mathrm{Fe}_{x} \mathrm{O}_{3}$ series. Its end members $\mathrm{YFeO}_{3}$ and $\mathrm{YCrO}_{3}$ both crystallize in the orthorhombic structure described by centrosymmetric space group Pnma. Although many efforts have been made to understand the magnetism and electric properties of these two materials, their multiferroic properties are still being debated [13-16]. Inspired by studies of $\mathrm{YBaCuFeO}_{5}$ and $\mathrm{Ca}_{2} \mathrm{Fe}_{2-x} \mathrm{Al}_{x} \mathrm{O}_{5}$ where

*michel.kenzelmann@psi.ch it was shown that site disorder can induce local frustration $[17,18]$, we studied the effects of mixing $\mathrm{YCrO}_{3}$ and $\mathrm{YFeO}_{3}$. The idea is that site disorder can induce local frustration, because it can locally frustrate magnetic correlations by introducing single-ion anisotropy disorder or exchange bond disorder. Nontrivial effects such as complex magnetic structures as well as ferroelectricity may result from the site disorder. A larger ferroelectric polarization, and a higher transition temperature, can be expected if ferroelectric polarization does not rely on spin-orbit interactions, but instead results from the local frustration of the dominant magnetic exchange in a material.

Indeed, in 2012, Rajeswaran et al. reported that upon substitution of $\mathrm{Fe}$ for $\mathrm{Cr}$ in $\mathrm{YCrO}_{3}$, one obtains materials that are described by space group Pnma and a dielectric anomaly near $T_{N}$ was observed, indicating a magnetodielectric effect [19]. In particular, they also provided evidence that $\mathrm{YCr}_{0.5} \mathrm{Fe}_{0.5} \mathrm{O}_{3}$ exhibits switchable electric polarization below $T_{N}=260 \mathrm{~K}$. The fact that the polarization occurs below $T_{N}$ suggests that ferroelectricity may have a magnetic origin, and the authors argued that the ferroelectric polarization in $\mathrm{YCr}_{0.5} \mathrm{Fe}_{0.5} \mathrm{O}_{3}$ could arise from the nonequivalent disordered spins at the $B$ site of the perovskite structure. Another study for $\mathrm{YCr}_{0.5} \mathrm{Fe}_{0.5} \mathrm{O}_{3}$ claimed the observation of dielectric relaxor behavior at around $500 \mathrm{~K}$ and $P-E$ hysteresis loop at room temperature [20].

To better understand the multiferroicity of $\mathrm{YCr}_{0.5} \mathrm{Fe}_{0.5} \mathrm{O}_{3}$ based on microscopic symmetry properties, we performed neutron powder diffraction (NPD) measurements on $\mathrm{YCr}_{0.5} \mathrm{Fe}_{0.5} \mathrm{O}_{3}$ to determine the magnetic ordering and its symmetry properties. Our NPD results show that $\mathrm{YCr}_{0.5} \mathrm{Fe}_{0.5} \mathrm{O}_{3}$ possesses a centrosymmetric crystal structure and a centrosymmetric magnetic structure for temperatures down to $T=2 \mathrm{~K}$, which is incompatible with the presence of magnetically induced ferroelectric polarization. In order to clarify the origin of ferroelectricity reported in this material, we also report a detailed analysis of the permittivity and the pyroelectric properties. We find no evidence for intrinsic ferroelectricity and instead associate our observations with 
the presence of defects in the samples. We propose that such defects contribute to the pyroelectric current through thermally stimulated currents due to the trapped charges at grain boundaries and possible local dipoles generated by the presence of oxygen vacancies.

\section{EXPERIMENTAL DETAILS}

High-quality polycrystalline samples of $\mathrm{YCr}_{0.5} \mathrm{Fe}_{0.5} \mathrm{O}_{3}$ were prepared by solid-state reaction as described in detail in Ref. [19]. Neutron powder diffraction measurements were carried out using the high-resolution diffractometer HRPT and high-intensity diffractometer DMC at the Swiss Spallation Neutron Source (SINQ), Paul Scherrer Institute. The neutron wavelengths we used for HRPT and DMC measurements were $\lambda=1.8857 \AA$ and $\lambda=4.507 \AA$, respectively. The NPD data were analyzed using the Rietveld package FULLPROF SUITE [21]. The temperature $(T)$ and magnetic-field $(H)$ dependent DC magnetization $(M)$ data were collected by using a superconducting quantum interference device magnetometer (Quantum Design MPMS-XL). The field-cooling (FC) mode was used to measure the susceptibility with an applied magnetic field of $H=0.01 T$ in the temperature range between 2 to $400 \mathrm{~K}$. The dielectric constant was measured by using an Agilent-4980A LCR meter at different frequencies on a hard disk-shaped pellet $1.8 \mathrm{~mm}$ in diameter and $0.22 \mathrm{~mm}$ in thickness. Silver paste was applied as electrodes. The same sample was used to measure pyroelectric current with a Keithley 6517B electrometer. The sample was first poled with an electric field $E=3.33 \mathrm{kV} / \mathrm{cm}$ from 300 or 50 to $2 \mathrm{~K}$, and after a wait of half an hour, measurements were taken while warming the sample from 2 to $300 \mathrm{~K}$.

\section{RESULTS AND DISCUSSION}

To characterize the magnetic properties of $\mathrm{YCr}_{0.5} \mathrm{Fe}_{0.5} \mathrm{O}_{3}$, $T$ - and $H$-dependent DC magnetization $(M)$ measurements were performed. In Fig. 1(a), we show the temperaturedependent magnetization $(M-T)$ of $\mathrm{YCr}_{0.5} \mathrm{Fe}_{0.5} \mathrm{O}_{3}$ under $\mathrm{FC}$ condition with an applied field of $H=100 \mathrm{Oe}$. The observed behavior characterizes the antiferromagnetic ordering below $T_{N}=260 \mathrm{~K}$. Figure 1 (b) presents the magnetization versus magnetic-field $(M-H)$ curves of $\mathrm{YCr}_{0.5} \mathrm{Fe}_{0.5} \mathrm{O}_{3}$ collected between 5 and $300 \mathrm{~K}$. At $5 \mathrm{~K}$, the curve displays hysteretic behavior, together with a dominant linear contribution, showing that the magnetic structure features a small ferromagnetic component. With increasing the temperature, the hysteresis loop gets suppressed gradually and disappears between 170 and $230 \mathrm{~K}$. For temperatures above $230 \mathrm{~K}$, linear behavior is observed as a function of field. These results are consistent with the previous reports $[19,22,23,32]$. The $M-T$ curve also features a magnetization reversal at around $250 \mathrm{~K}$, which was also observed in other studies. Efforts have been made to understand the magnetization reversal in $\mathrm{YCr}_{0.5} \mathrm{Fe}_{0.5} \mathrm{O}_{3}$, and it was argued that it may arise from the presence of the isotropic superexchange interactions and the antisymmetric Dzyaloshinskii-Moriya (DM) interactions [22-24]. Our current studies do not shed additional light on its origin.

To determine the magnetic ordering and symmetry properties of $\mathrm{YCr}_{0.5} \mathrm{Fe}_{0.5} \mathrm{O}_{3}$, we performed a series of neutron
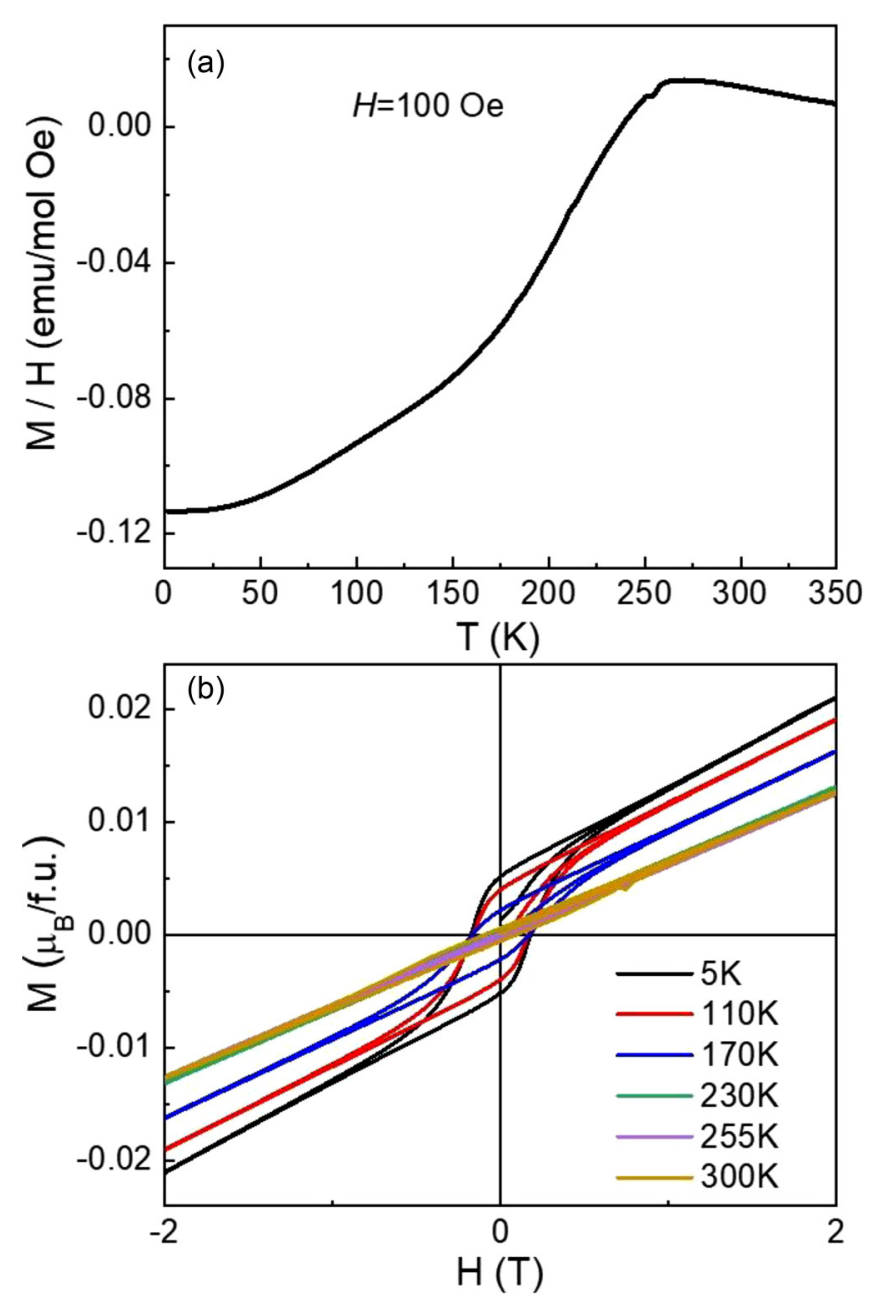

FIG. 1. Temperature-dependent magnetic susceptibility (a) and $M-H$ measurements at representative temperatures (b).

powder diffraction measurements both on HRPT and DMC. Figure 2(a) shows the diffraction pattern together with the Rietveld refinement results at $T=2 \mathrm{~K}$. The Rietveld analysis of the crystal structure illustrates that $\mathrm{YCr}_{0.5} \mathrm{Fe}_{0.5} \mathrm{O}_{3}$ crystallizes in an orthorhombic structure with centrosymmetric space group Pnma. Variables in the refinements include the lattice constants $a, b, c$; the fractional coordinates of $\mathrm{Y} x, z$; $\mathrm{O} 1 x, z$; $\mathrm{O} 2 x, y, z$, and the isotropic thermal parameters $B_{i s o}$. The structural parameters obtained by refinement are listed in Table I. For $T=2 \mathrm{~K}$, where magnetic ordering exists, Fig. 1(a) shows a fit describing both the atomic and magnetic diffraction intensities. (Detailed information about the magnetic model is given below.) Low values of $R_{\mathrm{wp}}=5.39 \%, R_{\mathrm{p}}=4.50 \%$, and $\chi^{2}=3.14$ indicate a good agreement between the measured data and the fitted model. No impurities can be indexed in the measured pattern, confirming the good quality of the sample.

Figure 3(a) presents the temperature dependence of the NPD patterns which focus on magnetic diffraction measured on DMC from 2 to $270 \mathrm{~K}$. Long-range magnetic ordering becomes visible mainly represented by Bragg peaks at (110) and (011) below $T_{N}=260 \mathrm{~K}$. At $270 \mathrm{~K}$, we also observed some signals from the magnetic diffraction, which we attribute to areas with a slightly higher Fe concentration and a higher $T_{N}$. With decreasing the temperature further, the 


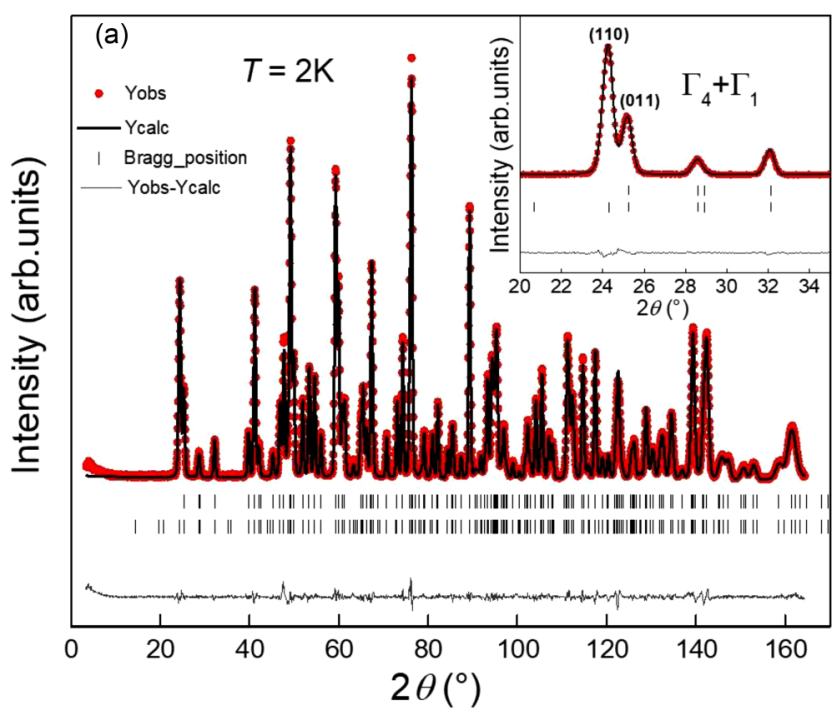

(b)

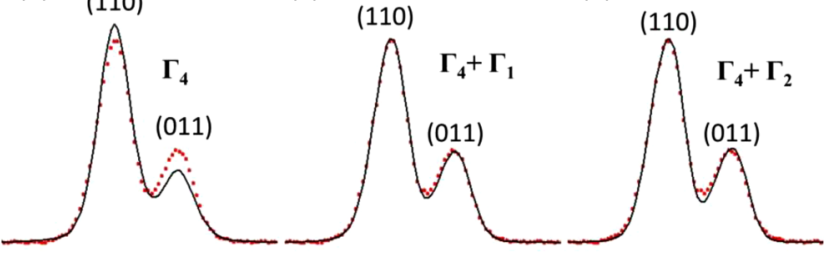

FIG. 2. (a) NPD pattern and Rietveld refinement result at $2 \mathrm{~K}$. Observed (red circle), calculated (black line), and difference (gray line) profiles are shown together with the allowed Bragg reflections of nuclear (top ticks), and magnetic (bottom ticks) diffraction. The inset shows the goodness of fitting for low-angle magnetic Bragg peaks. (b)-(d) Two fitted magnetic Bragg peaks, compared to the three different models.

magnetic Bragg peaks become stronger, but there are no new peaks or peak splitting down to $T=2 \mathrm{~K}$. The DMC measurements indicate a single type of magnetic ordering for
TABLE I. Crystallographic parameters of $\mathrm{YCr}_{0.5} \mathrm{Fe}_{0.5} \mathrm{O}_{3}$ at $2 \mathrm{~K}$ refined from NPD patterns.

\begin{tabular}{lcccccc}
\hline \hline Atoms & Site & $x$ & $y$ & $z$ & $g$ & Biso \\
\hline $\mathrm{Y}$ & $4 c$ & $0.0682(1)$ & $1 / 4$ & $-0.0178(1)$ & 1 & $0.181(13)$ \\
$\mathrm{Cr}$ & $4 b$ & 0 & 0 & $1 / 2$ & 0.5 & $0.068(18)$ \\
$\mathrm{Fe}$ & $4 b$ & 0 & 0 & $1 / 2$ & 0.5 & $0.068(18)$ \\
$\mathrm{O} 1$ & $4 c$ & $0.4617(1)$ & $1 / 4$ & $0.1087(2)$ & 1 & $0.288(17)$ \\
$\mathrm{O} 2$ & $8 d$ & $0.3027(1)$ & $0.0557(0)$ & $-0.3076(1)$ & 2 & $0.258(14)$ \\
\hline \hline
\end{tabular}

Space group Pnma (No. 62), a = 5.55429(8) $\mathrm{A}, \quad \mathbf{b}=7.55746$ (10) $\AA, \mathrm{c}=5.25431(6) \AA, R$ values $(\%): R_{\mathrm{wp}}=5.39, R_{\mathrm{p}}=4.50$; $\chi^{2}=3.14$.

temperatures between $T_{N}=260$ and $2 \mathrm{~K}$. Figures 3(b) and 3 (c) show the temperature-dependent intensity of (110) and (011), indicating a gradual weakening of the magnetic Bragg peaks with increasing temperature.

We now discuss the magnetic structure and symmetry properties of $\mathrm{YCr}_{0.5} \mathrm{Fe}_{0.5} \mathrm{O}_{3}$ at $T=2 \mathrm{~K}$. Before discussing the magnetic structure of $\mathrm{YCr}_{0.5} \mathrm{Fe}_{0.5} \mathrm{O}_{3}$, we review the magnetic structures of $\mathrm{YCrO}_{3}$ and $\mathrm{YFeO}_{3}$. Both $\mathrm{YCrO}_{3}$ and $\mathrm{YFeO}_{3}$ have commensurate spin ordering with $\mathbf{k}=(0,0,0)$ [25]. Studies indicate that both $\mathrm{YFeO}_{3}$ and $\mathrm{YCrO}_{3}$ order according to irreducible representation (irrep) $\Gamma_{4}\left(A_{x}, F_{y}, G_{z}\right)$, following the Bertaut notation [25,26]. With a vanishing magnetic component along the $a$ axis, both $\mathrm{YFeO}_{3}$ and $\mathrm{YCrO}_{3}$ have antiferromagnetically coupled magnetic moments $\boldsymbol{M}_{\boldsymbol{c}}$ along the $c$ axis with weak ferromagnetic component $\boldsymbol{M}_{\boldsymbol{b}}$ along the $b$ axis.

For $\mathrm{YCr}_{0.5} \mathrm{Fe}_{0.5} \mathrm{O}_{3}$, LeBail fitting for the magnetic diffraction reveals a commensurate magnetic order with wave vector $\mathbf{k}=(0,0,0)$. For $\mathbf{k}=(0,0,0)$, the little group $G_{\mathbf{k}}$ is identical to the space group Pnma and contains the symmetry elements $1,2_{x}, 2_{y}, 2_{z},-1, m_{x y}, m_{x z}, m_{y z}$. The magnetic unit cell of $\mathrm{YCr}_{0.5} \mathrm{Fe}_{0.5} \mathrm{O}_{3}$ possesses a single orbit with four magnetic sites inside, $\mathrm{Cr} / \mathrm{Fe} \_1(0,0,0.5), \mathrm{Cr} / \mathrm{Fe} \_2(0.5,0$,
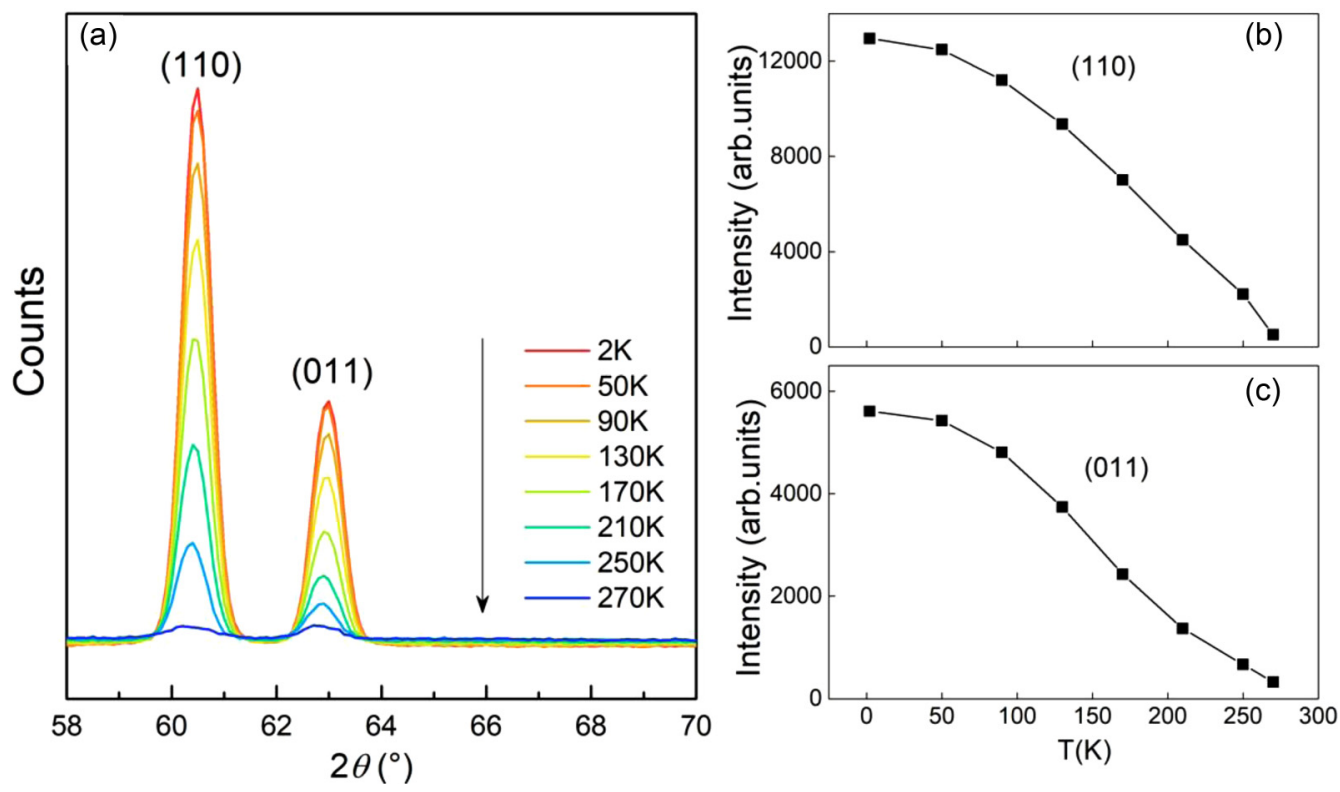

FIG. 3. (a) Representative NPD patterns collected at different temperatures on DMC. Temperature-dependent integrated intensity of (110) (b) and (011) (c). 
TABLE II. Character table of the little group $G_{\mathbf{k}}$ (top), basis vectors of irreps $\Gamma_{1}, \Gamma_{2}, \Gamma_{3}, \Gamma_{4}$ (middle) and agreement factors of fitting models $\Gamma_{4}, \Gamma_{4}+\Gamma_{1}, \Gamma_{4}+\Gamma_{2}$ (bottom).

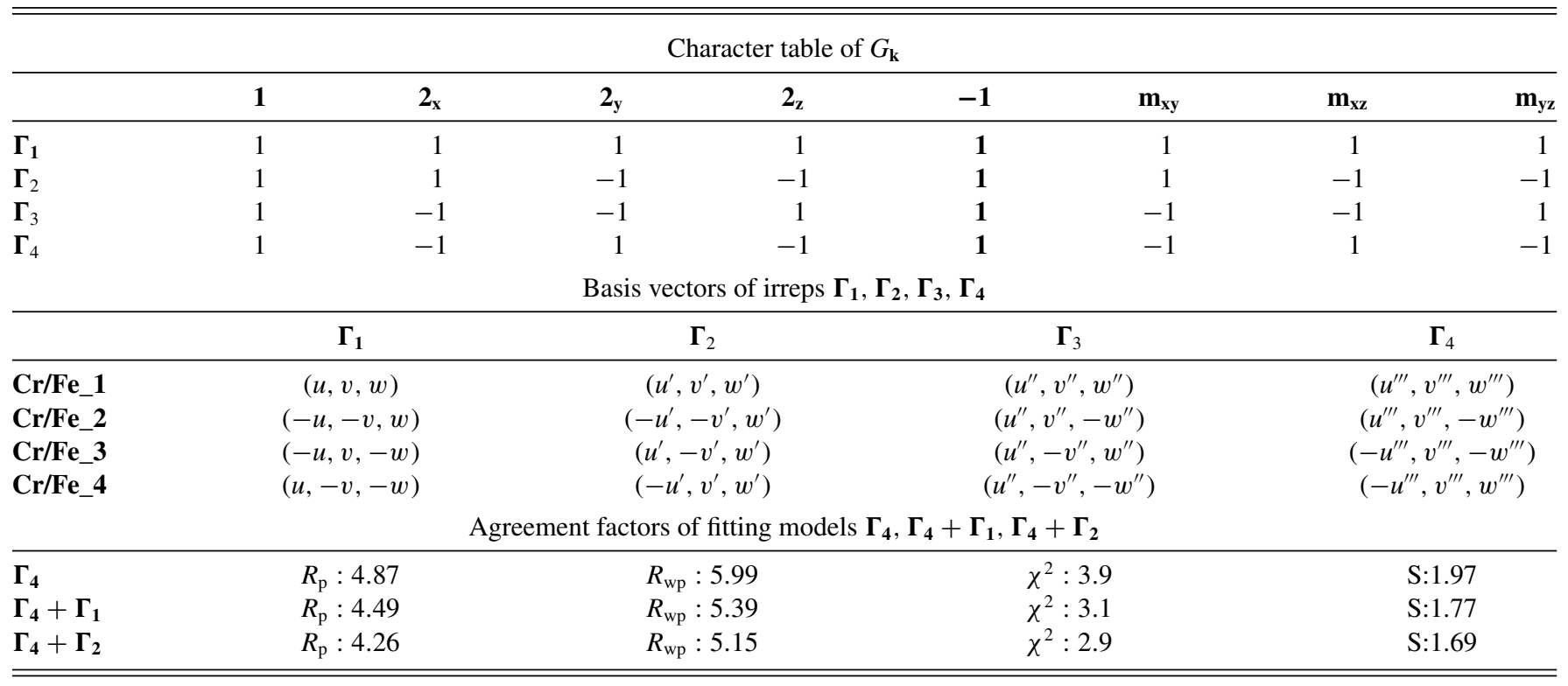

1), $\mathrm{Cr} / \mathrm{Fe} \_3(0,0.5,-0.5), \mathrm{Cr} / \mathrm{Fe} \_4(0.5,0.5,0)$. The $G_{\mathbf{k}}$ of $\mathbf{k}=(0,0,0)$ yields four irreps $\Gamma_{1}, \Gamma_{2}, \Gamma_{3}$, and $\Gamma_{4}$ for $\mathrm{Fe} / \mathrm{Cr}$ ordering. Table II shows the characters and the basis vectors of four irreps calculated by BASIREPS. Following Bertaut notation, the symmetry properties of the irreps are described by $\Gamma_{1}$ $\left(G_{x}, C_{y}, A_{z}\right), \Gamma_{2}\left(C_{x}, G_{y}, F_{z}\right), \Gamma_{3}\left(F_{x}, A_{y}, C_{z}\right), \Gamma_{4}\left(A_{x}, F_{y}, G_{z}\right)$. As shown in Table II, the space-inversion operation -1 always appears with character 1 , which means the inversion leaves the magnetic moments of $\mathrm{Cr} / \mathrm{Fe}$ invariant, confirming centrosymmetric magnetic structure of $\mathrm{YCr}_{0.5} \mathrm{Fe}_{0.5} \mathrm{O}_{3}$. Actually, the $4 b$ site of $\mathrm{Fe} / \mathrm{Cr}$ located at $(0,0,0.5)$ in Pnma is invariant under inversion at $(0,0,0)$. Due to the special symmetry, any possible irrep for this site leaves the spins invariant under the inversion operation and generates a centrosymmetric structure. As mentioned above, both time-inversion and space-inversion symmetry breaking are necessary for multiferroic materials. Our results thus rule out the possibility of spin-induced ferroelectricity in $\mathrm{YCr}_{0.5} \mathrm{Fe}_{0.5} \mathrm{O}_{3}$ or other $\mathrm{YMO}_{3}(M: 3 d$ transition metals) systems with a magnetic structure described by $\mathbf{k}=$ $(0,0,0)$. A group theoretical analysis on $\mathrm{SmFeO}_{3}$ yields the same conclusion that the magnetic ordering originating from Fe ions located at inversion centers cannot induce an electric polarization [27].

The magnetic structure of $\mathrm{YCr}_{0.5} \mathrm{Fe}_{0.5} \mathrm{O}_{3}$ was determined from both the HRPT and DMC data. As shown in Fig. 2, the two strongest magnetic Bragg peaks (110) and (011) in the pattern indicate that the magnetic moments point probably along the $c$ axis. Based on our calculation, the magnetic structure factors of (110) and (011) both feature $\left(+S_{1}-\right.$ $S_{2}-S_{3}+S_{4}$ ), where $S$ indicates the spin of $\mathrm{Cr} / \mathrm{Fe}$. To generate nonzero intensity and fit the magnetic diffraction, spin coupling with $\left(+S_{1}-S_{2}-S_{3}+S_{4}\right)$ along the $a, b$, or $c$ axis therefore should be considered. On the basis of the Rietveld refinement at $2 \mathrm{~K}$ with a single irrep, the best fit with $\chi^{2}=3.9$ was obtained for a structure described by $\Gamma_{4}$ with refined $\boldsymbol{M}_{\boldsymbol{c}}$ with antiferromagnetic coupling. Ferromagnetic coupled $\boldsymbol{M}_{\boldsymbol{b}}$ described by $\Gamma_{4}$ should be responsible for the magnetic hysteresis observed in the $M-H$ measurement, but it is too small to be determined by the refinement and was thus fixed to the value obtained from the $M-H$ measurements. Although $\Gamma_{4}$ can generally explain the magnetic diffraction, Fig. 2(b) shows clear intensity mismatches for both the (110) and (011) peaks with the current model. Fits using $\Gamma_{4}\left(\boldsymbol{M}_{\boldsymbol{c}}\right)+\Gamma_{1}\left(\boldsymbol{M}_{\boldsymbol{a}}\right)$ or $\Gamma_{4}\left(\boldsymbol{M}_{\boldsymbol{c}}\right)+\Gamma_{2}\left(\boldsymbol{M}_{\boldsymbol{a}}, \boldsymbol{M}_{\boldsymbol{b}}\right)$ pairs lead to better agreement factors, as shown at the bottom of Table II. Figures 2(b)-2(d) show the comparison of three different structure models $\Gamma_{4}, \Gamma_{4}$ $+\Gamma_{1}$, and $\Gamma_{4}+\Gamma_{2}$. Adding antiferromagnetically coupled $\boldsymbol{M}_{\boldsymbol{a}}$ described by $\Gamma_{1}$, model $\Gamma_{4}+\Gamma_{1}$ obviously improves the fit. The $\Gamma_{4}+\Gamma_{2}$ model implies competing antiferromagnetic and ferromagnetic components along the $b$ axis. We note that the competition of an antiferromagnetic and ferromagnetic component on the same site and along the same direction would involve competing interactions that are present neither in $\mathrm{YCrO}_{3}$ nor $\mathrm{YFeO}_{3}$. If these were indeed present in $\mathrm{YCr}_{0.5} \mathrm{Fe}_{0.5} \mathrm{O}_{3}$, they would thus be induced by site disorder, which is an interesting and unresolved aspect. Our current fits based on the powder-diffraction data cannot clearly distinguish the models $\Gamma_{4}+\Gamma_{1}$ and $\Gamma_{4}+\Gamma_{2}$, and single crystals are needed to unambiguously determine the magnetic structure.

Based on our refinement at $2 \mathrm{~K}$ using the $\Gamma_{4}+\Gamma_{1}$ model, the magnetic moment components along the crystallographic axes in units of $\mu_{\mathrm{B}}$ are $\boldsymbol{M}_{\boldsymbol{a}}=1.28(2), \boldsymbol{M}_{\boldsymbol{c}}=3.12(1)$. Refinement of the DMC data gives almost identical results. Figure 4 shows the magnetic structure obtained by our refinement together with the inversion center in the lattice.

In order to determine a possible subtle distortion and lower symmetry of the crystal structure in $\mathrm{YCr}_{0.5} \mathrm{Fe}_{0.5} \mathrm{O}_{3}$ and relate that to the reported ferroelectric polarization, we also performed neutron powder diffraction on HRPT with shorter wavelength $\lambda=1.49 \AA$ at $T=2 \mathrm{~K}$. By using a shorter wavelength, more diffraction peaks can be collected. This helps to identify lattice distortions which are better visible at high scattering angles. Polar subgroups Pmc21, Pmn21, and Pna21 of Pnma were considered for the refinement, but no 


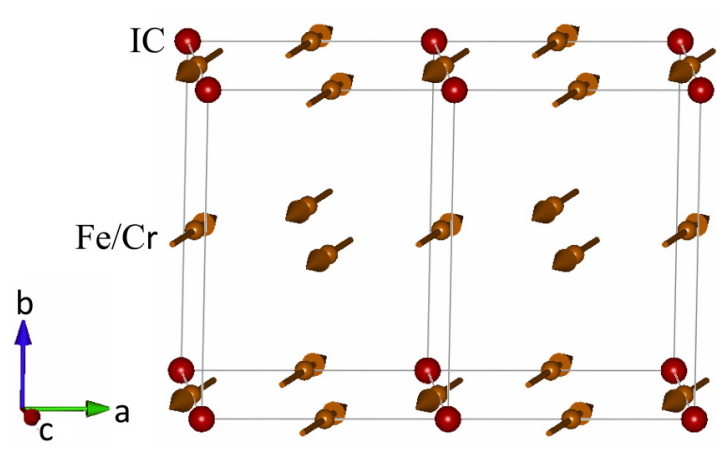

FIG. 4. Magnetic structure of $\mathrm{YCr}_{0.5} \mathrm{Fe}_{0.5} \mathrm{O}_{3}$ below $T_{N}$. $\mathrm{Cr} / \mathrm{Fe}$ magnetic moment (golden arrow) is shown together with the inversion center (red ball).

evidence of a symmetry lowering can be confirmed within the current resolution of the instrument. We thus claim both a centrosymmetric crystal structure and a centrosymmetric magnetic structure of $\mathrm{YCr}_{0.5} \mathrm{Fe}_{0.5} \mathrm{O}_{3}$.

To clarify the origin of electric polarization observed in Ref. [19], we carried out detailed investigations on the dielectric and pyroelectric properties. Figure 5 shows the temperature-dependent dielectric constant $\varepsilon(T)$ of $\mathrm{YCr}_{0.5} \mathrm{Fe}_{0.5} \mathrm{O}_{3}$ measured at different frequencies. The dielectric constant is almost independent of temperature up to $T=250 \mathrm{~K}$, above which it increases rapidly. No apparent anomalies could be detected in $\varepsilon(T)$ around the magnetic ordering temperature $T_{N}=260 \mathrm{~K}$. If an intrinsic second-order ferroelectric transition occurred at $T_{N}$, an anomaly should be observable in the measured frequency range, but none is observed. To study the effects of magnetic field on dielectric properties, we measured the temperature-dependent dielectric constant with $f=1 \mathrm{MHz}$ under $H=0.5 \mathrm{~T}$ (see inset of Fig. 5). No field-induced anomaly can be detected in the temperature range from 100 to $300 \mathrm{~K}$ which covers the magnetic ordering temperature $T_{N}=260 \mathrm{~K}$.

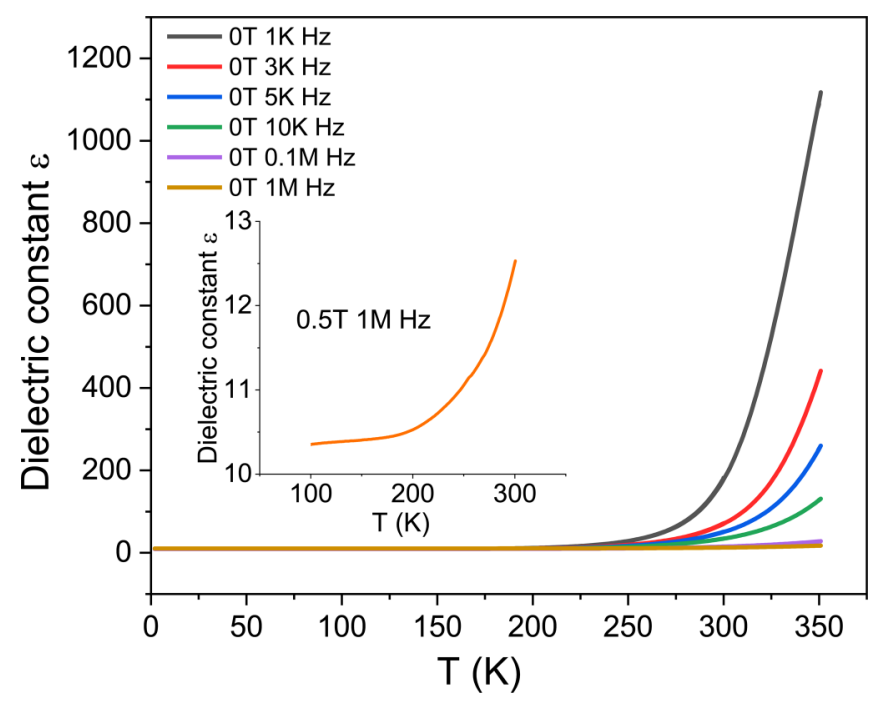

FIG. 5. Temperature-dependent dielectric constant $\varepsilon$ at different testing frequencies. The inset shows the result under magnetic field $H=0.5 \mathrm{~T}$.
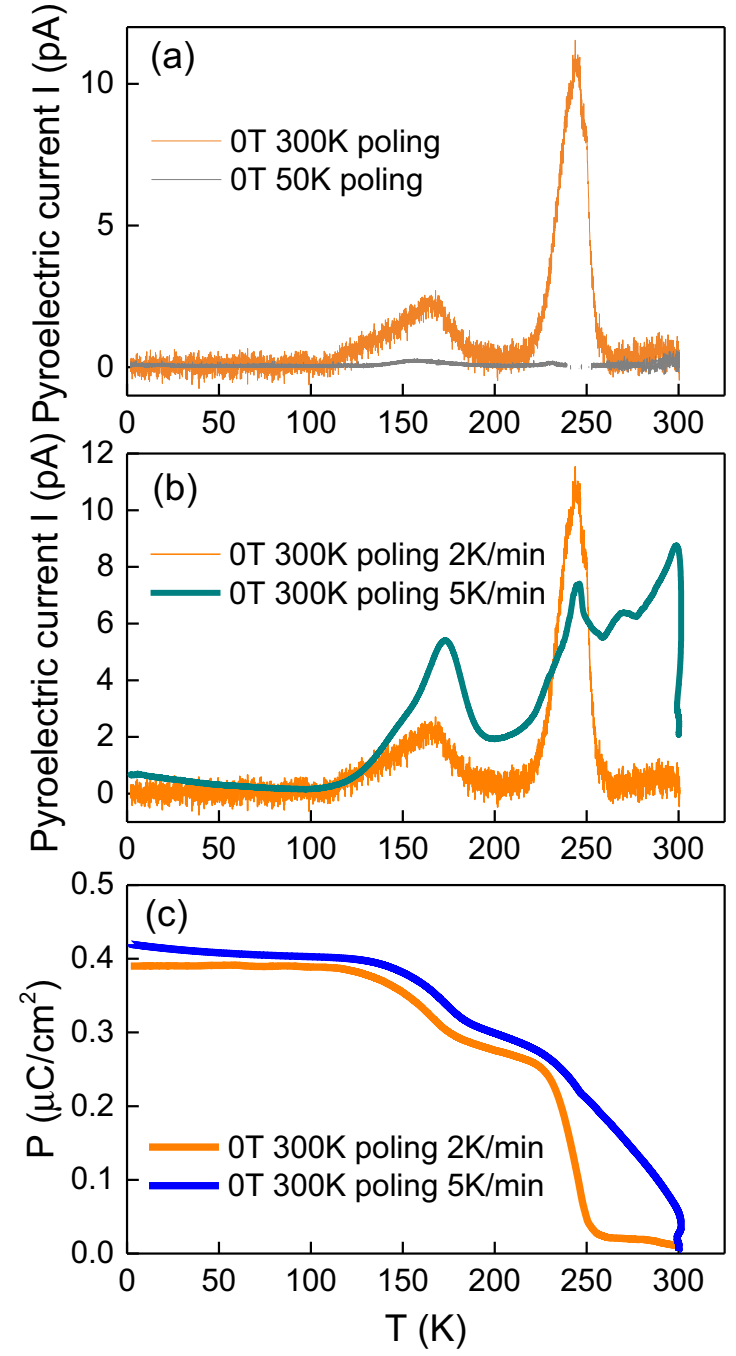

FIG. 6. Pyroelectric characterization with a poling electric field $E=3.33 \mathrm{kV} / \mathrm{cm}$ at different conditions (a), (b) and temperaturedependent electric polarization $P$ obtained by integrating the pyroelectric current (c).

We found that $\varepsilon(T)$ increases gradually with decreasing frequency and shows significant enhancement at $f=1 \mathrm{kHz}$. This behavior may be accounted for by the Debye relaxation in the frequency space or Maxwell-Wagner effect due to defects (grain boundaries, etc.) in the polycrystalline samples that give rise to an internal barrier layer capacitor [28]. At high frequencies, charge carriers do not have time to respond to the field, so that the measured capacitance (dielectric constant) is relatively small. At low frequencies, on the other hand, the charge carriers have time to follow the field, leading to an increased capacitance. For such a scenario, it is typical that the dielectric constant $\varepsilon$ is enhanced in the frequency region of about $f<1 \mathrm{kHz}$, which is what we observe as shown in Fig. 5.

We also searched for electric polarization by using pyroelectric measurements. The pyroelectric measurements were performed under different poling conditions and warming rates. The results are summarized in Fig. 6. In order to easily compare with previous studies in Ref. [19], we chose the applied voltage $E=3.33 \mathrm{kV} / \mathrm{cm}$ for all measurements. 
After poling the sample from 300 and $50 \mathrm{~K}$, respectively, to $2 \mathrm{~K}$, with $E=3.33 \mathrm{kV} / \mathrm{cm}$, pyroelectric current $I$ was measured while warming the sample at $2 \mathrm{~K} / \mathrm{min}$ from $T=2$ to $300 \mathrm{~K}$. As shown in Fig. 6(a), the pyroelectric current poling starting from $300 \mathrm{~K}$ shows two obvious anomalies. One strong feature is a symmetric peak that starts at about $T=225 \mathrm{~K}$ and extends to $260 \mathrm{~K}$, where long-range magnetic ordering emerges. Another anomaly sets in near $T=100 \mathrm{~K}$ and forms a weaker peak centered at $T \sim 170 \mathrm{~K}$ with asymmetric shape. In comparison, no visible pyroelectric current anomaly is found after poling from $50 \mathrm{~K}$. Figure $6(\mathrm{~b})$ presents the pyroelectric currents measured with heating speed 2 and $5 \mathrm{~K} / \mathrm{min}$. With larger heating rate $5 \mathrm{~K} / \mathrm{min}$, the lowtemperature pyroelectric current peak located at $T \sim 170 \mathrm{~K}$ shifts slightly to higher temperature. More pronounced are the effects for the high-temperature peak close to $T_{N}$ which changes greatly both the peak position and the peak shape, suggesting the presence of thermal relaxation process in $\mathrm{YCr}_{0.5} \mathrm{Fe}_{0.5} \mathrm{O}_{3}$.

We note however, that we obtained temperature-dependent polarization $P$ which is of the same order of magnitude as in Ref. [19]. In Fig. 6(c), we show the polarization $P$ derived by integrating the pyroelectric current measured in Fig. 6(b).

For intrinsic ferroelectric polarization, the pyroelectric current typically features a $\lambda$-like peak with narrow peak width due to the second-order ferroelectric phase transition. In the studies of $\mathrm{YCr}_{0.5} \mathrm{Fe}_{0.5} \mathrm{O}_{3}$, broad, symmetric peaks are observed which strongly depend on the heating rates and poling procedures. The pyroelectric currents that we observed share similarities but also look different from the results reported in Ref. [19], showing an obvious sample dependence. Also, with the dielectric measurement results in mind, we therefore conclude there is no support for intrinsic ferroelectricity in $\mathrm{YCr}_{0.5} \mathrm{Fe}_{0.5} \mathrm{O}_{3}$, and that the observed pyroelectric current may have a different origin.

We attribute the pyroelectric currents observed in our measurements and Ref. [19] to thermally stimulated currents which may arise from charges trapped at grain boundaries during the cooling under applied electric field [29,30]. It is known that in some materials, the thermally stimulated current leads to large electric currents during pyroelectric current measurements, and this has sometimes been misinterpreted as intrinsic ferroelectricity [31]. It is typical that thermally stimulated current generates relatively broad and symmetric peaks in the pyroelectric current, similar to what is observed in $\mathrm{YCr}_{0.5} \mathrm{Fe}_{0.5} \mathrm{O}_{3}$.

In addition, Fig. 6(a) shows that almost no pyroelectric current is observed after poling from $T=50 \mathrm{~K}$. Given that the poling electric field $E=3.33 \mathrm{kV}$ is sufficient to fully switch the electric polarization as shown in Ref. [19], the electric current peak in pyroelectric measurements should be observed even for poling from $T=50 \mathrm{~K}$ if it originated from ferroelectricity. However, our measurements show that the pyroelectric current strongly depends on the poling temperature $\left(T_{\text {pole }}\right)$. This is also consistent with thermally stimulated currents which are expected to show strong dependence on the value of $T_{\text {pole }}$.

Although the dielectric and pyroelectric measurements do not support intrinsic ferroelectric polarization in $\mathrm{YCr}_{0.5} \mathrm{Fe}_{0.5} \mathrm{O}_{3}$, it is confirmed by both our studies and
Ref. [19] that the pyroelectric current peak is located close to $T_{N}$. Further, there is evidence that the transition temperature varies with $\mathrm{Fe}$ content, indicating a connection between the magnetic ordering and the pyroelectric current. To understand this, we propose an alternative mechanism that is related to the oxygen vacancies in the system. In general, oxygen vacancies may be generated by the loss of oxygen at low oxygen partial pressure or during sintering at high temperatures. Oxygen vacancies induce valence-state variations of the cations as well as the formation of $\mathrm{Fe}^{2+} / \mathrm{Cr}^{2+}-\mathrm{Fe}^{3+} / \mathrm{Cr}^{3+}$ local dipoles. One may argue that the observed pyroelectric currents arise from a combined effect of the superexchange interactions and the local dipoles induced by the oxygen vacancies. Switching of the dipoles occurs through a transfer of electrons between $\mathrm{Fe}^{2+} / \mathrm{Cr}^{2+}$ and $\mathrm{Fe}^{3+} / \mathrm{Cr}^{3+}$ via the intermediate $\mathrm{O}$. Such an electron-transfer process may be influenced by the superexchange and DM interactions through the slight change of the angle between $\mathrm{Fe}^{2+} / \mathrm{Cr}^{2+}$ and $\mathrm{Fe}^{3+} / \mathrm{Cr}^{3+}$ at the onset of magnetic ordering. In fact, studies for $\mathrm{YFe}_{1-x} \mathrm{Cr}_{x} \mathrm{O}_{3}$ by $\mathrm{X}$-ray absorption near-edge spectroscopy measurements indicate the mixed-valence states of $\mathrm{Cr}$ and $\mathrm{Fe}$ ions in the lattice [32]. X-ray photoelectron spectrometry and Mössbauer spectral studies on $\mathrm{YFeO}_{3}$ single crystal also support such a scenario [14].

Therefore, the pyroelectric current may include contributions from both the trapped charges at the grain boundaries and the dipoles due to the oxygen vacancies. Besides $\mathrm{YCr}_{0.5} \mathrm{Fe}_{0.5} \mathrm{O}_{3}$ in our work, the complex electric properties observed in $\mathrm{YCrO}_{3}, \mathrm{YFeO}_{3}, \mathrm{YFe}_{0.8} \mathrm{Mn}_{0.2} \mathrm{O}_{3}$, and $\mathrm{SmFeO}_{3}$ may have a similar origin. Clean, high-quality single crystals with fully occupied $\mathrm{O}$ sites would be helpful to clarify the nature of the electric polarization in these systems.

\section{SUMMARY AND CONCLUSIONS}

This work investigated the magnetic ordering and the electric properties of $\mathrm{YCr}_{0.5} \mathrm{Fe}_{0.5} \mathrm{O}_{3}$ by neutron powder diffraction, dielectric, and pyroelectric measurements in the temperature range from 2 to $300 \mathrm{~K}$. $\mathrm{YCr}_{0.5} \mathrm{Fe}_{0.5} \mathrm{O}_{3}$ crystallizes in orthorhombic structure with space group Pnma. Rietveld analysis of the neutron powder diffraction indicates both the crystal structure and the magnetic structure of $\mathrm{YCr}_{0.5} \mathrm{Fe}_{0.5} \mathrm{O}_{3}$ are centrosymmetric below $T_{N}=260 \mathrm{~K}$. The magnetic structure can thus not give rise to the spin-induced ferroelectricity. Detailed dielectric and pyroelectric studies on $\mathrm{YCr}_{0.5} \mathrm{Fe}_{0.5} \mathrm{O}_{3}$ also do not support an intrinsic magnetically induced ferroelectricity but may be understood by processes related to the possible defects in the polycrystalline samples.

\section{ACKNOWLEDGMENTS}

This work is supported by the Swiss National Science Foundation under Grant No. 200021_165855. Y.W.L. was supported by the National Key R\&D Program of China (Grant No. 2018YFA0305700), and the NSFC (Grants No. 11574378 and No. 51772324). Neutron-scattering experiments were carried out at the continuous spallation neutron source SINQ at the Paul Scherrer Institut at Villigen PSI in Switzerland. 
[1] W. Erenstein, N. D. Mathur, and J. F. Scott, Nature (London) 442, 759 (2006).

[2] S. W. Cheong and M. Mostovoy, Nat. Mater. 6, 13 (2007).

[3] D. I. Khomskii, J. Magn. Magn. Mater. 306, 1 (2006).

[4] R. Ramesh, A. Nicola, and A. Spaldin, Nat. Mater. 6, 21 (2007).

[5] T. Kimura, T. Goto, H. Shintani, K. Ishizaka1, T. Arima, and Y. Tokura, Nature (London) 426, 55, (2003).

[6] K. Taniguchi, N. Abe, T. Takenobu, Y. Iwasa, and T. Arima, Phys. Rev. Lett. 97, 097203 (2006).

[7] D. Khomskii, Physics 2, 20 (2009).

[8] M. Kenzelmann, A. B. Harris, S. Jonas, C. Broholm, J. Schefer, S. B. Kim, C. L. Zhang, S. W. Cheong, O. P. Vajk, and J. W. Lynn, Phys. Rev. Lett. 95, 087206 (2005).

[9] J. Wang, J. B. Neaton, H. Zheng, V. Nagarajan, S. B. Ogale, B. Liu, D. Viehland, V. Vaithyanathan, D. G. Schlom, U. V. Waghmare, N. A. Spaldin, K. M. Rabe, M. Wuttig, and R. Ramesh, Science 299, 1719 (2003).

[10] X. Wang, Y. S. Chai, L. Zhou, H. B. Cao, C. Cruz, J. Y. Yang, J. H. Dai, Y. Y. Yin, Z. Yuan, S. J. Zhang, R. Z. Yu, M. Azuma, Y. Shimakawa, H. M. Zhang, S. Dong, Y. Sun, C. Q. Jin, and Y. W. Long, Phys. Rev. Lett. 115, 087601 (2015).

[11] L. Zhou, J. H. Dai, Y. S. Chai, H. M. Zhang, S. Dong, H. B. Cao, S. Calder, Y. Y. Yin, X. Wang, X. D. Shen, Z. H. Liu, T. Saito, Y. Shimakawa, H. Hojo, Y. Ikuhara, M. Azuma, Z. W. Hu, Y. Sun, C. Q. Jin, and Y. W. Long, Adv. Mater. 29, 1703435 (2017).

[12] G. Lawes, A. B. Harris, T. Kimura, N. Rogado, R. J. Cava, A. Aharony, O. Entin-Wohlman, T. Yildrim, M. Kenzelmann, C. Broholm, and A. P. Ramirez, Phys. Rev. Lett. 95, 087205 (2005).

[13] M. Y. Shang, C. Y. Zhang, T. S. Zhang, L. Yuan, L. Ge, H. M. Yuan, and S. H. Feng, Appl. Phys. Lett. 102, 062903 (2013).

[14] Z. X. Cheng, H. Shen, J. Y. Xu, P. Liu, S. J. Zhang, J. L. Wang, X. L. Wang, and S. X. Dou, J. Appl. Phys. 111, 034103 (2012).
[15] C. R. Serrao, A. K. Kundu, S. B. Krupanidhi, U. V. Waghmare, and C. N. R. Rao, Phys. Rev. B 72, 220101(R) (2005).

[16] K. Ramesha, A. Llobet, T. Proffen, C. R. Serrao, and C. N. R. Rao, J. Phys.:Condens. Matter 19, 102202 (2007).

[17] M. Morin, E. Canévet, A. Raynaud, M. Bartkowiak, D. Sheptyakov, V. Ban, M. Kenzelmann, E. Pomjakushina, K. Conder, and M. Medarde, Nat. Commun. 4, 2511 (2013).

[18] N. Abe, N. D. Khanh, T. Sasaki, and T. Arima, Phys. Rev. B 89, 054437 (2014).

[19] B. Rajeswaran, P. Mandal, R. Saha, E. Suard, A. Sundaresan, and C. N. R. Rao, Chem. Mater. 24, 3591 (2012).

[20] V. G. Nair, A. Das, V. Subramanian, and P. N. Santhosh, J. Appl. Phys. 113, 213907 (2013).

[21] J. Rodríguez-Carvajal, Physica B 192, 55 (1993).

[22] J. H. Mao, Y. Sui, X. Q. Zhang, Y. T. Su, X. J. Wang, Z. J. Liu, Y. Wang, R. B. Zhu, Y. Wang, W. F. Liu, and J. K. Tang, Appl. Phys. Lett. 98, 192510 (2011).

[23] N. Dasari1, P. Mandal, A. Sundaresan, and N. S. Vidhyadhiraja, EPL 99, 17008 (2012).

[24] O. V. Billoni, F. Pomiro, S. A. Cannas, C. Martin, A. Maignan, and R. E. Carbonio, J. Phys.:Condens. Matter 28, 476003 (2016).

[25] R. L. White, J. Appl. Phys. 40, 1061 (1969).

[26] A. K. Zvezdin and A. A. Mukhin, JETP Lett. 88, 505 (2008).

[27] R. D. Johnson, N. Terada, and P. G. Radaelli, Phys. Rev. Lett. 108, 219701 (2012).

[28] G. Catalana, Appl. Phys. Lett. 88, 102902 (2006).

[29] R. R. Haering and E. N. Adams, Phys. Rev. 117, 451 (1960).

[30] H. Okino, Y. Toyoda, M. Shimizu, T. Horiuchi, T. Shiosaki, and K. Matsushige, Jpn. J. Appl. Phys. 37, 5137 (1998).

[31] H. Nhalil, H. S. Nair, C. M. N. Kumar, A. M. Strydom, and S. Elizabeth, Phys. Rev. B 92, 214426 (2015).

[32] F. A. Fabian, P. P. Pedra, K. O. Moura, J. G. S. Duque, and C. T. Meneses, J. Magn. Magn. Mater. 408, 94 (2016). 